\title{
THE EXISTENCE OF INVARIANT SUBSPACES ${ }^{1}$
}

\author{
BY LOUIS DE BRANGES AND JAMES ROVNYAK \\ Communicated by P. R. Halmos, May 4, 1964
}

We have succeeded in showing that a bounded linear transformation in a Hilbert space always has invariant subspaces. The existence of invariant subspaces was previously known only under complete continuity hypotheses.

THEOREM. Let $T$ be a bounded linear transformation in a Hilbert space 3e. Let $f$ be a given element of $\Re$ and let $h$ be a given number,

$$
0 \leqq h \leqq\|T f\|^{2} .
$$

Then there exist projections $P_{+}$and $P_{-}$into invariant subspaces for $T$ such that the range of $P_{+}$contains the range of $P_{-}$, the orthogonal complement of the range of $P_{-}$in the range of $P_{+}$has dimension 0 or 1 , and

$$
\left\|T P_{-} f\right\|^{2} \leqq h \leqq\left\|T P_{+} f\right\|^{2} .
$$

The proof depends on Livsič's theory of characteristic operator functions [8], [9]. A characteristic operator function is an operator valued analytic function which is associated with the transformation in such a way that invariant subspaces for the transformation correspond to factors of the function. In general, this correspondence is only formal. Two technical problems have to be solved to show the existence of invariant subspaces. The first is to obtain factorization theorems without making a complete continuity hypothesis, and the second is to obtain an isometric inclusion for related Hilbert spaces. The first problem is solved by using a weak compactness theorem for positive definite operator valued functions. The second problem is solved by using functions which are analytic across the boundary of the unit disk. The theory is formulated in terms of formal power series rather than analytic functions.

We choose and fix a coefficient space. This is a Hilbert space $\mathfrak{e}$ that we treat as a generalization of the complex numbers. The elements of $\mathfrak{e}$ will be called vectors, and the bounded linear transformations of $\mathfrak{e}$ into itself will be called operators. The norm of a vector $c$ is written $|c|$. If $b$ is a vector, then $\bar{b}$ is the linear functional on $\mathfrak{e}$ such that $\bar{b} a=\langle a, b\rangle$ for every vector $a$. The adjoint and norm of an operator $A$ are denoted by $\bar{A}$ and $|A|$.

\footnotetext{
${ }^{1}$ Research supported by the Alfred P. Sloan Foundation and the National Science Foundation.
} 
Let $\mathfrak{C}(z)$ be the Hilbert space of formal power series $f(z)=\sum a_{n} z^{n}$, with vector coefficients, such that

$$
\|f\|^{2}=\sum\left|a_{n}\right|^{2}<\infty .
$$

Let $B(z)$ be a formal power series with operator coefficients. Suppose that when $g(z)$ is in $\mathcal{C}(z), B(z) g(z)$ is in $\mathcal{C}(z)$ and $\|B(z) g(z)\| \leqq\|g(z)\|$. An equivalent condition for this is that $B(z)$ converge and represent a function $B(w)$ which is bounded by 1 in the unit disk, $|w|<1$. If $f(z)$ is in $\mathrm{C}(z)$, let its $B$-norm be defined by

$$
\|f(z)\|_{B}^{2}=\sup \left[\|f(z)+B(z) g(z)\|^{2}-\|g(z)\|^{2}\right]
$$

where the supremum is taken over all $g(z)$ in $\mathcal{C}(z)$. Let $\mathfrak{F}(B)$ be the set of all $f(z)$ in $\mathfrak{e}(z)$ such that $\|f(z)\|_{B}<\infty$. Then $\mathcal{H}(B)$ is a Hilbert space in the $B$-norm. If $f(z)$ is in $\mathfrak{F C}(B)$, then $[f(z)-f(0)] / z$ is in $\mathcal{H C}(B)$ and

$$
\|[f(z)-f(0)] / z\|_{B}^{2} \leqq\|f(z)\|_{B}^{2}-|f(0)|^{2} .
$$

The general case of the spaces $\mathcal{F}(B)$ was introduced (in a different way) in the second author's thesis [12].

THEOREM 1. Let $T$ be a linear transformation in a Hilbert space Ke. Suppose that $\|T\| \leqq 1$ and $\lim \left\|T^{n} f\right\|=0$ for every $f$ in TC. Suppose also that the dimension of the range of $1-T^{*} T$ is no more than the dimension of $\mathcal{C}$. Then $T$ is unitarily equivalent to the transformation $f(z)$ $\rightarrow[f(z)-f(0)] / z$ in some space $\operatorname{FC}(B)$ which is contained isometrically in $\mathrm{e}(z)$.

Therefore the problem is to determine the spaces $\mathfrak{F}(A)$ which are contained isometrically in a given space $\mathfrak{H C}(B)$. This is related to a factorization problem.

Theorem 2. Let $\mathfrak{H C}(A)$ and $\mathfrak{H C}(B)$ be given spaces. A necessary and sufficient condition that $\mathfrak{H C}(A)$ be contained in $\mathfrak{F}(B)$ and that the inclusion not increase norms is that $B(z)=A(z) C(z)$ where the space $\mathfrak{H C}(C)$ exists.

When the inclusion fails to be isometric, it is because of an overlapping phenomenon. For simplicity, suppose that $\mathcal{H}(B)$ is contained isometrically in $\mathfrak{C}(z)$. The problem is to know when $\mathfrak{H C}(A)$ is contained isometrically in $\mathfrak{C}(z)$.

THEOREM 3. A necessary and sufficient condition that a space $\operatorname{3C}(B)$ be contained isometrically in $\mathrm{C}(z)$ is that $\mathrm{HC}(B)$ contain no nonzero element of the form $B(z) L(z)$, where $L(z)$ is in $\mathrm{e}(z)$. 
A similar situation arises in the study of non-selfadjoint transformations (de Branges [4]). There, a Hilbert space is constructed from the series which cause the trouble.

ThEOREM 4. Let $\Re(B)$ be a given space. Let $\mathbb{L}=\mathfrak{L}_{B}$ be the set of power series $L(z)$ in $\mathfrak{C}(z)$ such that $B(z) L(z)$ is in $\mathfrak{H}(B)$. Then $\&$ is a Hilbert space in the norm

$$
\|L(z)\|_{\mathcal{L}}^{2}=\|L(z)\|^{2}+\|B(z) L(z)\|_{B}^{2} .
$$

If $L(z)$ is in $\&$, then $[L(z)-L(0)] / z$ is in $\&$. The transformation $L(z)$ $\rightarrow[L(z)-L(0)] / z$ in $\&$ has an isometric adjoint.

If $\mathfrak{K}(B)$ is a given space, there is a positive definite function $K(\alpha, \beta)(|\alpha|<1$ and $|\beta|<1)$ which completely determines $\mathcal{H}(B)$.

THEOREM 5. Let $\mathfrak{H C}(B)$ be a given space. If $c$ is any vector and if $|w|<1$, then

$$
K(w, z) c=[1-B(z) \bar{B}(w)] c /(1-z \bar{w})
$$

belongs to $\mathfrak{H C}(B)$ as a power series in $z$, and $\bar{c} f(w)=\langle f(z), K(w, z) c\rangle_{B}$ holds for every $f(z)$ in $\mathfrak{H C}(B)$. If $\left(w_{i}\right)$ is any finite set of points in the unit disk, then

$$
\sum \bar{c}_{j} K\left(w_{i}, w_{j}\right) c_{i} \geqq 0
$$

for all corresponding choices of vectors $\left(c_{i}\right)$.

Compactness follows from a characterization of this positive definite function.

Theorem 6. Let $\left(\mathfrak{H C}\left(B_{n}\right)\right)$ be any sequence of spaces. If the coefficient space is separable, there is a subsequence $\left(\mathfrak{H C}\left(B_{n(k)}\right)\right)$ and a space $\mathfrak{F}(B)$ such that $\bar{c} K(\alpha, \beta) c=\lim \bar{c} K_{n(k)}(\alpha, \beta) c$ for every vector $c$ when $|\alpha|<1$ and $|\beta|<1$.

A study of finite-dimensional $\mathfrak{H C}(B)$ spaces now leads to the factorization theorem.

THEOREM 7. Let $\mathfrak{H C}(B)$ be a given space. Let $c$ be a vector and let $h$ be a number,

$$
0 \leqq h \leqq|c|^{2}-|\bar{B}(0) c|^{2} .
$$

If the coefficient space is separable, there exist spaces $\mathfrak{H C}(A), \mathfrak{F}(S)$, and $\mathfrak{H C}(C)$ such that $B(z)=A(z) S(z) C(z), \mathfrak{F}(S)$ has dimension 0 or 1 , and

$$
|c|^{2}-|\bar{A}(0) c|^{2} \leqq h \leqq|c|^{2}-|\bar{S}(0) \bar{A}(0) c|^{2} .
$$


A special choice of $B(z)$ is needed to convert this factorization theorem into the existence theorem for invariant subspaces.

Theorem 8. Let $\operatorname{FC}(B)$ be a given space. Suppose that $\operatorname{HC}(B)$ is contained isometrically in $\mathrm{C}(\mathrm{z})$. Suppose that the series which belong to $\mathfrak{H}(B)$ converge and represent functions in a disk $|w|<a$ where $a>1$, and that the transformation $f(z) \rightarrow f(w)$ takes $\mathfrak{H C}(B)$ continuously into $\mathfrak{C}$ when $|w|<a$. Then $\mathfrak{H C}(B)$ is equal isometrically to a space $\mathfrak{H C}(A)$, where $A(z)$ converges and represents a function $A(w)$ in the disk $|w|<a$, and $A(w)$ has unitary values on the circle $|w|=1$.

A complete account will be published elsewhere.

Added in proof. R. G. Douglas has discovered a gap in the argument as originally given. To fill it make the following additional hypothesis in Theorem 7 and in the necessity for Theorem 2: multiplication by $B(z)$ is isometric in $\mathcal{C}(z)$. The main theorem is unchanged.

\section{REFERENCES}

1. N. Aronszajn and K. T. Smith, Invariant subspaces of completely continuous operators, Ann. of Math. (2) 60 (1954), 345-350.

2. A. Beurling, On two problems concerning linear transformations in Hilbert space, Acta Math. 81 (1949), 239-255.

3. L. de Branges, Some Hilbert spaces of analytic functions. I, Trans. Amer. Math. Soc. 106 (1963), 445-468.

4. - Some Hilbert spaces of analytic functions. II, J. Math. Anal. Appl. (to appear).

5. - Some Hilbert spaces of analytic functions. III, J. Math. Anal. Appl. (to appear).

6. M. S. BrodskiY and M. S. Livsix, Spectral analysis of non-selfadjoint operators and intermediate systems, Uspehi Mat. Nauk 13 (79) (1958), 3-85. (Russian)=Amer. Math. Soc. Transl. (2) 13 (1960), 265-346.

7. P. R. Halmos, Shifts on Hilbert spaces, J. Reine Angew. Math. 208 (1961), 102112.

8. M. S. Livsic, On a class of linear operators in Hilbert space, Mat. Sb. (N.S.) 19 (61) (1946), 239-262. (Russian)

9. - On the spectral decomposition of a linear non-selfadjoint operator, Mat. Sb. (N.S.) 34 (76) (1954), 145-199. (Russian)=Amer. Math. Soc. Transl. (2) 5 (1957), 67-114.

10. J. Rovnyak, Ideals of square summable power series, Proc. Amer. Math. Soc. 13 (1962), 360-365.

11. - Ideals of square summable power series. II, Proc. Amer. Math. Soc. (to appear).

12. - Some Hilbert spaces of analytic functions, Thesis, Yale University, New Haven, Conn., 1963.

Purdue University 